\title{
AIP
}

\section{Dissipative structures in the CO oxidation on $\mathrm{Pt}(100)$}

R. F. S. Andrade, D. Lima, G. Dewel, and P. Borckmans

Citation: J. Chem. Phys. 100, 9192 (1994); doi: 10.1063/1.466674

View online: http://dx.doi.org/10.1063/1.466674

View Table of Contents: http://jcp.aip.org/resource/1/JCPSA6/v100/i12

Published by the AIP Publishing LLC.

Additional information on J. Chem. Phys.

Journal Homepage: http://jcp.aip.org/

Journal Information: http://jcp.aip.org/about/about_the_journal

Top downloads: http://jcp.aip.org/features/most_downloaded

Information for Authors: http://jcp.aip.org/authors

\section{ADVERTISEMENT}

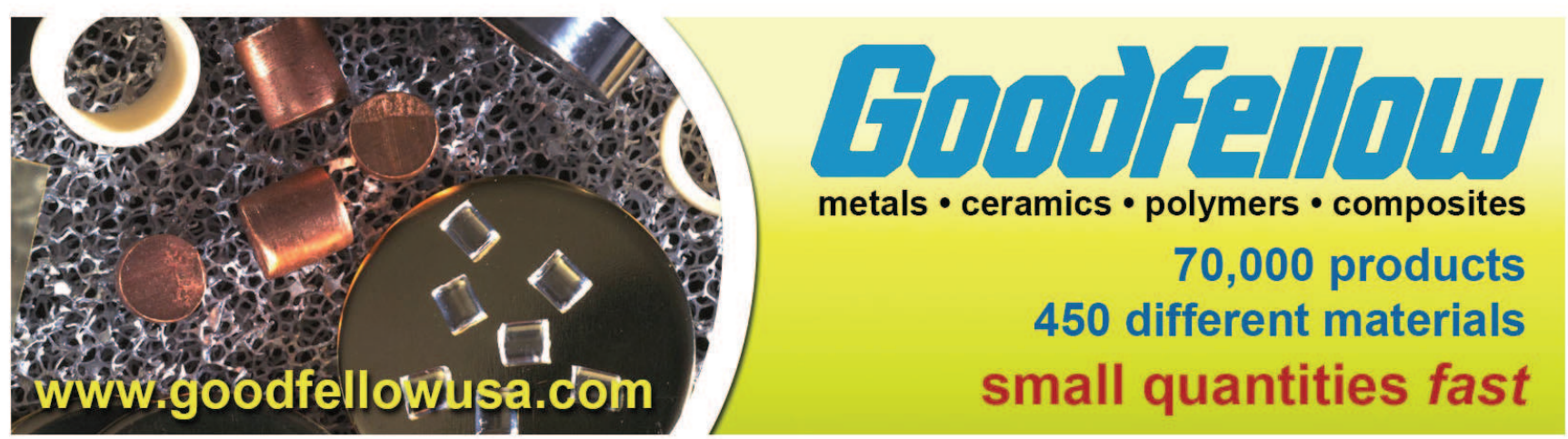




\title{
Dissipative structures in the $\mathrm{CO}$ oxidation on $\mathrm{Pt}(100)$
}

\author{
R. F. S. Andrade and D. Lima ${ }^{\text {a) }}$ \\ Instituto de Física, Universidade Federal da Bahia, 40210-Salvador, Brazil \\ G. Dewel $\left.\right|^{\text {b) }}$ and P. Borckmans ${ }^{\text {b) }}$ \\ Service de Chimie-Physique, Center for Nonlinear Phenomena and Complex Systems, CP 231, \\ Université Libre de Bruxelles, 1050-Bruxelles, Belgium
}

(Received 25 February 1993; accepted 31 January 1994)

\begin{abstract}
A model for the homogeneous kinetic oscillations in the $\mathrm{CO}$ oxidation on $\mathrm{Pt}(100)$ is extended to describe space dependent situations by the introduction of two diffusive-like processes. As is well known these increase the size of the instability domain. Depending on the values of the diffusion coefficients, they lead to the existence, when the surface is homogeneous, of stationary periodic space patterns besides the uniform oscillating solutions. This may be shown both theoretically through linear stability analysis or numerical computation.
\end{abstract}

\section{INTRODUCTION}

The observation of dissipative structures ${ }^{1}$ (temporal, spatial, or spatiotemporal) in catalytic reactions has been the subject of intense research in past years. ${ }^{2-5}$ They have been observed in a large variety of systems operating in very different regimes. The $\mathrm{CO}$ oxidation on $\mathrm{Pt}$ is one of the simplest catalytic systems where these phenomena are observed. Due to the simplicity of the reaction mechanism, the investigation of this system is important to uncover the basic steps which lead to the oscillations ${ }^{6}$ and pattern selection.

In the case of $\mathrm{Pt}$ monocrystals under ultrahigh vacuum conditions, temporal oscillations of the reaction products have been observed when the crystalline surfaces (100) or (110) act as catalysts. Several surface analyzing techniques (LEED, work function, etc.) have shown that these oscillations are correlated with periodic changes of the crystalline surface state: ${ }^{7-9} 1 \times 1$ and hex phases on Pt(100) and $1 \times 1$ and $1 \times 2$ phases on $\mathrm{Pt}(110)$. Moreover, the use of scanning LEED procedures, ${ }^{10}$ and more recently by scanning photoemission microscopy (SPM) ${ }^{11}$ and photoemission electron microscopy $(\mathrm{PEEM})^{12}$ has shown the presence of spatia ${ }^{13}$ and spatiotemporal dissipative structures. ${ }^{14}$ Thus it is now well settled that, despite the particular properties of these different Pt surfaces, the interaction between the crystalline structure of the metal surface and the chemical kinetics is at the heart of the mechanism which generates the observed oscillations and structures.

The LEED studies of the CO oxidation on $\mathrm{Pt}(100)$ and $\mathrm{Pt}(110)$ have indicated different behavior for the two different surfaces: Spatial coupling through the gas phase usually leads to quite regular kinetic oscillations on $\mathrm{Pt}(110)$, at least at high temperatures. ${ }^{9}$ On the other hand, various scanning studies clearly revealed that the oscillations on $\mathrm{Pt}(100)$ are associated with the propagation of reaction fronts. ${ }^{710,15}$ These waves are generally triggered at surface imperfections such as structured defects yielding irregular temporal behav-

\footnotetext{
a) Supported by CAPES-CNPq (Brazil).

${ }^{b)}$ Research Associate with the F.N.R.S. (Belgium).
}

ior. From the theoretical side, the modeling of this reaction using cellular automata techniques have shown that they could also be formed on a perfectly uniform surface, ${ }^{16}$ although the investigation of the same problem by a set of reaction-diffusion equations ${ }^{17}$ detected the presence of wave fronts only when trigger zones were present.

In this work we pursue previous investigations ${ }^{18,19}$ we have performed on the homogeneous $\mathrm{CO}$ oxidation on $\mathrm{Pt}(100)$ to include an analysis of spatiotemporal dissipative structures. We use the site interaction model to explore novel aspects of the phase transitions in the crystalline structure of the surface, whereas the dynamical behavior is described by the system of differential equations, where two diffusion terms are now present. The paper is organized according to the following scheme: in Sec. II the homogeneous model is extended to treat space dependent situations. This amounts to reconsidering the site model used to derive the surface free energy and to include the extra energy cost for the presence of coexisting patches of different structure (interface energy). Our treatment points to the presence of one more diffusionlike term in the equations of motion, while other models consider only the ordinary diffusion of the adsorbed CO which is also included in the model. In Sec. III we present the linear stability analysis of the derived reaction-diffusion equations and construct the new instability region in the $P_{\mathrm{CO}} \times P_{\mathrm{O}_{2}}$ plane. This investigation also indicates what type of solution should appear when the steady-state solutions become unstable. In Sec. IV we discuss the solutions obtained from a numerical integration of the system of partial differential equations when the surface is assumed to be uniform. Stationary periodically modulated patterns, uniformly oscillating solutions, and mixed modes of these are obtained. In Sec. V we show that space dependent properties of the surface, expressed by different values of a model parameter which measures the rate of surface defects, gives rise to states with wave propagation. Finally Sec. VI closes the paper with an overall discussion of our results and concluding remarks. 


\section{SPACE DEPENDENT MODELS}

Let us briefly recall the main features of the previous model used in Ref. 19. The kinetics of the reaction follows the Langmuir-Hinshelwood mechanism on the catalytic active phase $(1 \times 1)$, where both $\mathrm{CO}$ and $\mathrm{O}_{2}$ adsorb and react. On the catalytic inert phase (hex) only $\mathrm{CO}$ adsorbs. Other mechanisms present in the system are the phase transitions and the trapping. The first one has been conveniently described by a Ginzburg-Landau potential, from which the equation of motion for the surface state variable can be derived. The other step refers to the migration of $\mathrm{CO}$ adsorbed on hex to a neighboring empty $1 \times 1$ site, leaving an empty hex site. This is a local mechanism and should not be considered as diffusion. The system of equations reads

$$
\begin{aligned}
\frac{d u_{a}}{d t}= & a k_{1} P_{\mathrm{CO}}-k_{2} u_{a}+k_{3} a u_{b}-k_{4} \frac{u_{a} v_{a}}{a}, \\
\frac{d u_{b}}{d t}= & (1-a) k_{1} P_{\mathrm{CO}}-k_{6} u_{b}-k_{3} a u_{b}, \\
\frac{d v_{a}}{d t}= & a k_{7} P_{\mathrm{O}_{2}}\left[\left(1-\frac{s v_{a}}{a}-\frac{r u_{a}}{a}\right)^{2}+\alpha\left(1-\frac{s v_{a}}{a}\right)^{2}\right] \\
& -k_{4} \frac{u_{a} v_{a}}{a}, \\
\frac{d a}{d t}= & k_{8}\left[\frac{u_{a}+v_{a}}{a}-\bar{\eta}_{2} a-\bar{\eta}_{3} a^{2}-\bar{\eta}_{4} a^{3}\right] .
\end{aligned}
$$

$u_{a}, u_{b}$, and $v_{a}$ are, respectively, the fractions of sites occupied by $\mathrm{CO}$ on $1 \times 1$ and hex sites and of $\mathrm{O}_{2}$ on $1 \times 1$; $a$ represents the fraction of $1 \times 1$ sites on the surface. The constants $k_{i}$ refer to the different basic steps of the systems. The $\bar{\eta}_{i}$ are related with the coefficients of the GinzburgLandau potential. $r$ and $s$ are related to the inhibition of $\mathrm{O}_{2}$ adsorption due to the presence of preadsorbed $\mathrm{CO}$ and $\mathrm{O}$. $\alpha$ is a parameter measuring the rate of surface defects and favors the inhibition of $\mathrm{O}_{2}$ adsorption.

The extension of this model to space dependent situations amounts to consider local, space dependent variables and diffusion-like terms in some of the equations of the system. The first aspect does not require any special modification. As to the diffusion terms, they originate from two different mechanisms: First there exists the normal surface diffusion of adsorbed species. However the dissociative adsorption of $\mathrm{O}_{2}$ leads to rather immobile $\mathrm{O}$ surface atoms. Thus following Imbihl et al. ${ }^{17}$ we consider only the surface diffusion of $\mathrm{CO}$, whose molecules are loosely bonded to the surface, and also neglect the diffusion of $\mathrm{CO}$ in the hex phase. Therefore, changes must be introduced only in Eq. (1), which becomes

$$
\frac{\partial u_{a}}{\partial t}=a k_{1} P_{\mathrm{CO}}-k_{2} u_{a}+k_{3} a u_{b}-k_{4} \frac{u_{a} v_{a}}{a}+k_{5} \nabla^{2} u_{a} .
$$

Second there is the mechanism of phase transitions between the two different crystalline arrangements of the surface atoms $(1 \times 1$ and hex), which is induced by the presence of adsorbates. Such phenomenon can be treated by the site interaction model introduced in Ref. 18, which was the base of the derivation of the Ginzburg-Landau surface free energy and Eq. (4). If we now consider that the variables are space dependent, we can show that the model gives rise to a diffusion-like term in Eq. (4). The model was based on the following Hamiltonian:

$$
H\left(\left\{\phi_{i}\right\}\right)=H^{1}\left(\left\{\phi_{i}\right\}\right)+H^{2}\left(\left\{\phi_{i}\right\}\right)+H^{3}\left(\left\{\phi_{i}\right\}\right),
$$

where $\phi_{i}$ is the site variable which may assume the discrete values $0,1 / 2$, or 1 according to whether the site is on the hex phase, on the border between hex and $1 \times 1$ patches, or on the $1 \times 1$ phase. The explicit expressions for the three terms in Eq. (5) are

$$
H^{1}\left(\left\{\phi_{i}\right\}\right)=\sum_{i} H_{i}^{1}\left(\phi_{i}\right) ; \quad H_{i}^{1}\left(\phi_{i}\right)= \begin{cases}0 & \text { if } \phi_{i}=0 \\ \gamma & \text { if } \phi_{i}=1 / 2 \\ \delta & \text { if } \phi_{i}=1\end{cases}
$$

$$
H^{2}\left(\left\{\phi_{i}\right\}\right)=\sum_{i} H_{i}^{2}\left(\phi_{i}, n_{i}\right)=\sum_{i}-n_{i}\left[\epsilon_{h}\left(1-\phi_{i}\right)+\epsilon_{1} \phi_{i}\right]
$$

$$
\begin{aligned}
H^{3}\left(\left\{\phi_{i}\right\}\right)=\sum_{(i, j)}^{*} H_{i j}^{3}\left(\phi_{i}, \phi_{j}\right)= & \sum_{(i, j)} \epsilon\left[\phi_{i}\left(\tau-\phi_{j}\right)\right. \\
& \left.+\phi_{j}\left(\tau-\phi_{i}\right)\right] .
\end{aligned}
$$

The $n_{i}$ 's are the occupation number of the $i$ th site; all other parameters refer to the different interaction energies of a given site to his neighbors and to adsorbed atoms and their precise meanings are discussed in Ref. 19.

Now we will show that a mean-field solution for the free energy of the model leads to an explicit dependence on the rate with which the mean value of the site variables changes along the surface. This procedure is well known in magnetism, leading, e.g., to the occurrence of walls between magnetic domains. ${ }^{20}$ For this purpose we write $\phi_{i}=\phi\left(\mathbf{r}_{i}\right)$, where $\mathbf{r}_{i}$ locates the $i$ th site on the surface. Now we assume that the value of $\phi(r)$ changes very slowly with the position, so that, for the neighboring sites $\mathbf{r}_{j}$, we may write

$\phi\left(\mathbf{r}_{j}\right) \simeq \phi\left(\mathbf{r}_{i}\right)+\left[\left(\mathbf{r}_{j}-\mathbf{r}_{i}\right) \cdot \nabla\right] \phi\left(\mathbf{r}_{i}\right)+\frac{1}{2}\left[\left(\mathbf{r}_{j}-\mathbf{r}_{i}\right) \cdot \nabla\right]^{2} \phi\left(\mathbf{r}_{i}\right)$.

Since the $\phi$ will explicitly depend on $r$, it is convenient to define a functional form for the Ginzburg-Landau potential:

$$
g_{\mathrm{GL}}=\int_{S} \rho\left[-\epsilon_{1} \theta_{a} a+\eta_{2} a^{2}+\eta_{3} a^{3}+\eta_{4} a^{4}+\frac{k_{9}^{\prime}}{2}(\nabla a)^{2}\right] d S .
$$

$S$ indicates the whole surface of the sample and $\rho$ the density of sites. The first four terms inside the integral were already present in Ref. 18, while the derivation of the last one will be presented below. Moreover the equation of the motion for the variable $a$ will be derived from

$$
\int_{S} \rho \frac{\partial a}{\partial t} d S=-k \frac{1}{\epsilon_{1}} \frac{\delta g_{\mathrm{GL}}}{\delta a(r)} .
$$

Now if we insert Eq. (9) into Eq. (8) we get, after some algebraic manipulations, the following expression: 


$$
\begin{aligned}
H^{3}\left(\left\{\phi_{i}\right\}\right) \simeq & \epsilon \sum_{i, j}\left\{\left[\tau-\phi\left(\mathbf{r}_{i}\right)\right] \phi\left(\mathbf{r}_{i}\right)+\frac{1}{4}\left[\tau-2 \phi\left(\mathbf{r}_{i}\right)\right]\right. \\
& \left.\times\left[\left(\mathbf{r}_{j}-\mathbf{r}_{i}\right) \cdot \nabla\right]^{2} \phi\left(\mathbf{r}_{i}\right)\right\},
\end{aligned}
$$

where the sum over pairs in Eq. (8) has been changed into a sum over lattice sites. We notice in Eq. (12) two different terms. The first one describes the energy for a homogeneous sample, i.e., when we put $\phi\left(\mathbf{r}_{i}\right)=\phi\left(\mathbf{r}_{j}\right)$. The second term depends on the way $\phi$ changes along the surface and is the relevant term for our discussion. So let us focus the attention on

$$
\begin{aligned}
& -\frac{\epsilon}{2} \sum_{i, j} \phi\left(\mathbf{r}_{i}\right)\left[\left(\mathbf{r}_{j}-\mathbf{r}_{i}\right) \cdot \nabla\right]^{2} \phi\left(\mathbf{r}_{i}\right) \\
& \simeq-\frac{\epsilon z c^{2}}{2} \sum_{i} \phi\left(\mathbf{r}_{i}\right) \nabla^{2} \phi\left(\mathbf{r}_{i}\right) \\
& \simeq-\frac{\epsilon z c^{2}}{2} \int_{S} \rho \phi\left(\mathbf{r}_{i}\right) \nabla^{2} \phi\left(\mathbf{r}_{i}\right) d S,
\end{aligned}
$$

where $z$ is the number of nearest neighbors, $c$ is the lattice spacing, and $\rho$ is the density of sites found on the catalysts' surface. If we now perform a partial integration of Eq. (13) it reduces to

$-\frac{\epsilon z c^{2} \rho}{2} \int_{S}\left|\nabla \phi\left(\mathbf{r}_{i}\right)\right|^{2} d S$.

Now we recognize in Eq. (14) the same form of the last term in Eq. (10), from which the value of $k_{9}^{\prime}$ can immediately be obtained. Moreover the variation of the functional $g_{\mathrm{GL}}$, as indicated in Eq. (11), will give rise to the following equation, which with Eqs. $\left(1^{\prime}\right),(2)$, and (3) constitute the basic system which will be considered in the next sections:

$$
\frac{\partial a}{\partial t}=k_{8}\left[\frac{u_{a}+v_{a}}{a}-\bar{\eta}_{2} a-\bar{\eta}_{3} a^{2}-\bar{\eta}_{4} a^{3}\right]+k_{9} \nabla^{2} a,
$$

where $k_{9}=k_{9}^{\prime} / \epsilon_{1}=z \epsilon c^{2} / \epsilon_{1}$.

The numerical value for the diffusion coefficient $k_{5}$ can be measured in experiments, ${ }^{21}$ whereby its order of magnitude is $10^{-7} \mathrm{~cm}^{2} / \mathrm{s}$. On the other hand the value of $k_{9}$ can be computed from the above expression, taking for $\epsilon$ and $\epsilon_{1}$ those values used in the site interaction model introduced in Ref. 19. It is seen that $k_{9}$ has an order of magnitude of $10^{-15}$ $\mathrm{cm}^{2} / \mathrm{s}$ which is much smaller than that of $k_{5}$. This difference justifies neglecting $k_{9}$ with respect to $k_{5}$ as done in Ref. 17 . However, the linear stability analysis shows that the instability diagram with respect to wave-like perturbations, together with the bifurcation type and the wave number of the unstable mode, depend strongly on the values of $k_{5}$ and $k_{9}$. So we will keep both diffusion terms in our system, and will dispose of their values to present different kinds of behavior.

The linear stability analysis carried out in Ref. 19 for the fixed points of $S 1$ shows, for the values of the constants shown in Table $I$, the existence of two negative eigenvalues with very large absolute value $\left(\lambda_{1} \simeq-10^{5} \simeq-k_{4}\right.$. and $\lambda_{2} \simeq-10^{2} \simeq-k_{6}-a k_{3}$ ). Therefore, the dynamics along the
TABLE I. Parameter values.

\begin{tabular}{cl}
\hline \hline Constant & Value in this work \\
\hline$k_{1}$ & $2.5 \times 10^{5} \mathrm{ML} \mathrm{s}^{-1} \mathrm{Torr}^{-1}$ \\
$k_{2}$ & $1 \mathrm{~s}^{-1}$ \\
$k_{3}$ & $50 \mathrm{~s}^{-1}$ \\
$k_{4}$ & $10^{2}-10^{5} \mathrm{ML}^{-1} \mathrm{Torr}^{-1}$ \\
$k_{5}$ & $10^{-4}-10^{-3} \mathrm{~cm}^{2} \mathrm{~s}^{-1}$ \\
$k_{6}$ & $10 \mathrm{~s}^{-1}$ \\
$k_{7}$ & $5.6 \times 10^{4} \mathrm{ML} \mathrm{s}^{-1} \mathrm{Torr}^{-1}$ \\
$k_{8}$ & $1 \mathrm{~s}^{-1}$ \\
$k_{9}$ & $10^{-4}-10^{-1} \mathrm{~cm}^{2} \mathrm{~s}^{-1}$ \\
$\bar{\eta}_{2}$ & 2 \\
$\bar{\eta}_{3}$ & -10 \\
$\bar{\eta}_{4}$ & 7 \\
$r$ & 2 \\
$s$ & $5 / 3$ \\
$\alpha$ & $0.15-0.4$ \\
\hline \hline
\end{tabular}

direction of the respective eigenvectors are of a fast relaxing type, justifying the adiabatic elimination of these fast relaxing modes, and leading to the following two-dimensional model ( $S 2)$, which shows the same basic features of the complete four-dimensional one $(S 1)$ :

$$
\begin{aligned}
\frac{\partial w}{\partial t}= & a k_{1} P_{\mathrm{CO}} \frac{(1+q)}{(1+a q)}-k_{2} \frac{(w+|w|)}{2}+\Theta(w) k_{5} \nabla^{2} w \\
& -a k_{7} P_{\mathrm{O}_{2}}\left[\left(1-\frac{(r-s) w+(r+s)|w|}{2 a}\right)^{2}\right. \\
& \left.+\alpha\left(1-\frac{s(w-|w|)}{2 a}\right)^{2}\right], \\
\frac{\partial a}{\partial t}= & k_{8}\left[\frac{|w|}{a}-\bar{\eta}_{2} a-\bar{\eta}_{3} a^{2}-\bar{\eta}_{4} a^{3}\right]+k_{9} \nabla^{2} a
\end{aligned}
$$

The new variable $w$ introduced in the process of the adiabatic elimination is expressed in terms of the former variables as

$$
w=u_{a}-v_{a}+a q u_{b} /(1+a q)
$$

where $q=k_{3} / k_{6}$ and $\Theta(w)$ indicates the Heaviside function $[\Theta(w)=0$, if $w<0 ; \Theta(w)=1$, if $w>0]$. The two equations of $S 2$ are not exact, but, as we have indicated above, admit corrections on their right-hand side proportional to $O\left[1 /\left(k_{6}+a k_{3}\right)\right] \approx 10^{-2}$, if we consider the typical values for the reaction constants.

\section{LINEAR STABILITY ANALYSIS}

The fixed points corresponding to homogeneous situations of both systems $S 1$ and $S 2$ were discussed in Ref. 19 . They describe those homogeneous states where the amount of gases adsorbing and reacting does not depend upon the position on the catalyst's surface and is constant with time. The present linear stability analysis differs from that one as we now allow for perturbations of the type.

$$
\delta u_{a}=\delta u_{a}^{0} \exp [i(\kappa x-\omega t)]
$$




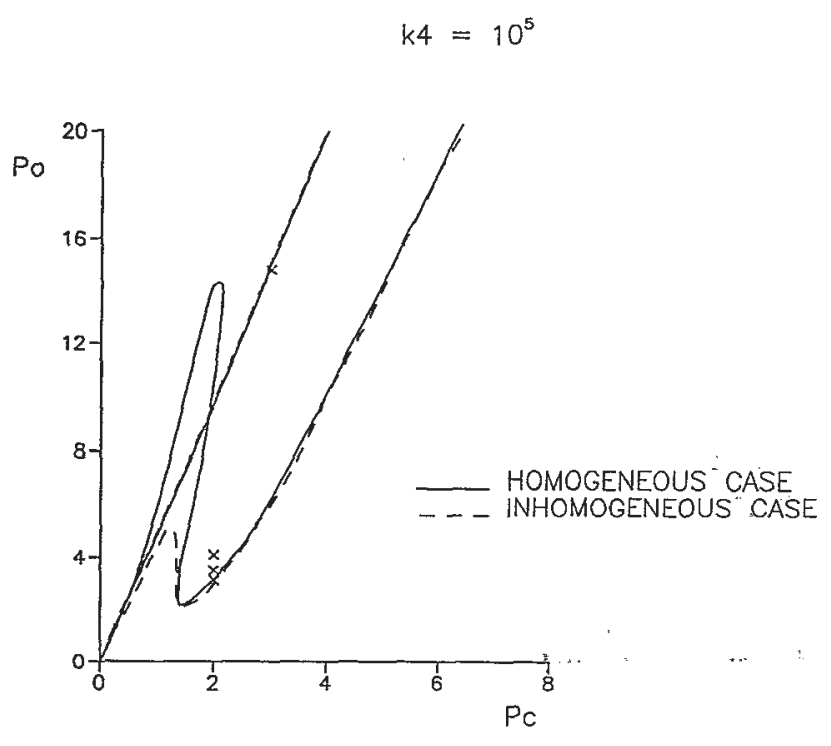

FIG. 1. Stability diagram in the (reduced) partial pressure plane. Only for small values of $P_{c}$ and $P_{o}$ the instability regions with respect to homogeneous and nonhomogeneous perturbations differ substantially.

where $\kappa$ is the wave number and $\omega$ the complex eigenfrequencies. The values for $\omega$, given by the secular equation, are $\kappa$ dependent and their imaginary parts control the stability of the corresponding fixed point with respect to a given perturbation. By assigning the values of the parameters where the imaginary parts of all $\omega$ 's are negative one can construct the stability region with respect to any possible real value of $\kappa$. The actual stability region is given by the intersection of the all $\kappa$ dependent stability regions.

The stability question depends upon the behavior of the two eigenvalues of the original model (S1) labeled as $\lambda_{3}$ and $\lambda_{4}$, which are almost the same [with corrections of order $1 /\left(k_{6}+a k_{3}\right)$ as the two eigenvalues of the reduced model $(S 2)] .{ }^{19}$ Depending upon the values of the partial pressures their imaginary parts can change sign, indicating a change in the stability of the fixed points (FP). The results we get working with either of the models are qualitatively the same, with corrections of the same order in the values of the partial pressures where the points become unstable.

The instability region, the type of bifurcation the system undergoes and the wavelength of the unstable mode, depend strongly on the values of the diffusion coefficients. We know from Ref. 19 that the homogeneous system looses its stability through a Hopf or a saddle-node bifurcation, while the latter may actually become of SNIPER type. To investigate the influence of the diffusion terms we consider the values of $P_{c}$ and $P_{o}$ for which the system $S 2$ lies on the transition line of the homogeneous system. Then we analyze, for the two types of bifurcation, the behavior of the eigenvalues $\lambda_{3,4}$ as a function of the wave number $\kappa$. It is clear that $\operatorname{Re}\left[\lambda_{i}(\kappa=0)\right]=0$, for at least one of the eigenvalues, say $\lambda_{3}$, which we will henceforth label as $\lambda$. When we let $\kappa$ increase, $\operatorname{Re}[\lambda(\kappa)]$ will be nonzero. We look for the existence of an interval of values of $\kappa$ within which $\operatorname{Re}(\lambda)>0$. If this is the case, then there will be a value $\kappa=\kappa_{\max }$, satisfying

$$
\left.\frac{d}{d \kappa} \cdot \operatorname{Re}(\lambda)\right|_{\kappa=\kappa_{\max }} ^{\mid}=0,
$$

which will be, up to corrections of higher order, the mode to first become unstable when we move in the parameter space towards the instability region. Indeed, this mode has become unstable prior to the homogeneous mode $(\kappa=0)$, and the emerging state will be space modulated. If $\operatorname{Re}[\lambda(\kappa \neq 0)$ ]$<0 \forall \kappa$, the homogeneous mode is the first one to become unstable, and the bifurcation will occur without space modulation.

Our investigation follows the following steps: First obtain the values of $\kappa_{\max }$, solutions of Eq. (19). If all of them are complex, there is no space modulated instability. If there exists real $\kappa_{\max }$, space modulated instabilities are conditioned to $\operatorname{Re}\left[\lambda\left(\kappa_{\max }\right)\right]>0$. The expressions for $\kappa_{\max }^{2}$ when the bifurcation of the homogeneous system is of Hopf type and saddle node are, respectively, given by

$$
\begin{aligned}
\kappa_{\max }^{2}= & {\left[2 m_{11} \pm\left(k_{5}+k_{9}\right) \sqrt{-m_{12} m_{21} / k_{5} k_{9}}\right] /\left(k_{5}-k_{9}\right), } \\
\kappa_{\max }^{2}= & {\left[m_{11}-m_{22} \pm\left(k_{5}+k_{9}\right) \sqrt{-m_{11} m_{22} / k_{5} k_{9}}\right] / } \\
& \left(k_{5}-k_{9}\right) .
\end{aligned}
$$

In the above expressions $m_{i j}$ note the matrix elements of the homogeneous problem. To decide about the type of bifurcation we have to consider each point on the transition line, since the $m_{i j}$ will depend upon the values of the partial pressures. The overall features of our investigation may be summarized as follows: space dependent instabilities will occur only when $k_{5} / k_{9}<0.3$. For the actual values of $k_{5}$ and $k_{9}$ $\left(k_{5} / k_{9} \gg 1\right)$, the linear stability indicates no changes either in

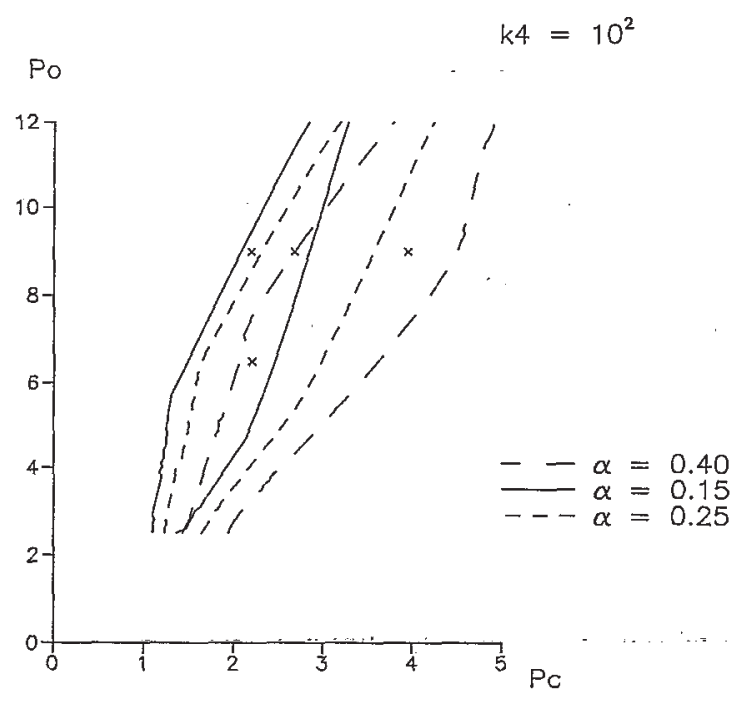

FIG. 2. Stability diagram for different values of $k_{4}$ and $\alpha$. Note the strong dependence of the form of the instability region on $\alpha$. The values of $k_{4}$ has little influence. 

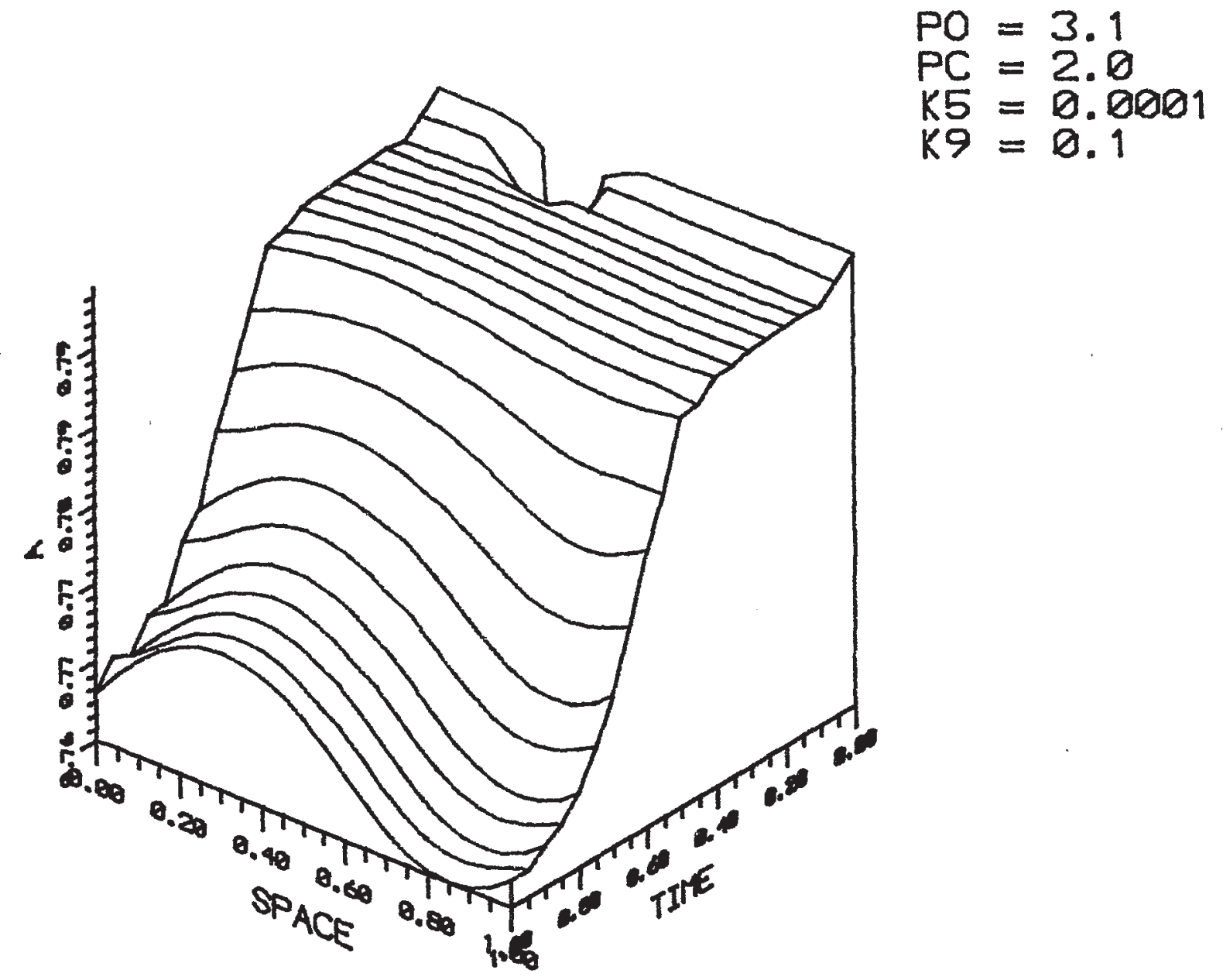

FIG. 3. Surface time-space evolution of the variable $a$, when $\left(P_{c}, P_{o}\right)=(2,3.1)$. The length of the sample is $0.35 \mathrm{~cm}$ and the total time of integration is $50 \mathrm{seg}$.

the form of the instability region or in the type of bifurcation with respect to the results obtained for the homogeneous situation.

However we think it is of theoretical interest to investigate the main features of the space modulated modes which emerge when $k_{9}$ takes sufficiently large values. For the purpose of showing clearly this effect we consider, for instance, $k_{5}=10^{-4}$ and $k_{9}=0.1$. In Fig. 1 we draw the instability region in the $P_{o} \times P_{c}$ diagram, where $P_{o}=2 r k_{7} P_{\mathrm{o}_{2}}$ and $P_{c}=2 r k_{1} P_{\mathrm{CO}}$, which is characterized by the absence of any stable FP with respect to perturbations of the form (18). For the purpose of comparison we also draw the instability region obtained in Ref. 19 with respect to homogeneous perturbations. We note that, with the exception of the region at small pressures, the two stability regions almost overlap, although the new one is a little bit larger than the former, so that one may expect the occurrence of uncommon behaviors, resulting from the interaction of the two instabilities. As discussed above, if we are in the stable region and move in the parameter space $\left(p_{c}\right.$ or $\left.p_{o}\right)$ towards the region of instability, the first unstable state we meet is a nonhomogeneous state with a well defined value of the wave number $\kappa \neq 0$. Moreover, since the eigenvalue which becomes critical is a pure imaginary number, the state we reach is characterized by a stationary space periodic pattern, which is typical for the Turing transitions. As we will discuss in Sec. V, this kind of behavior predicted by the linear theory is also observed when we integrate the nonlinear equations. This analysis shows very clearly that this instability is not of the same kind as that one which causes the presence of wave fronts.

In Fig. 2 we show the stability diagrams of the homogeneous system for other values of the parameter $\alpha$ and the constant $k_{4}$. Their location in the $P_{c} \times P_{o}$ plane will be of relevance in the discussion of the emergence of traveling waves on the nonhomogeneous catalyst surface. We note that the shape and size of the instability regions are more sensitive to the value of $\alpha$ than to that of $k_{4}$ : indeed, for smaller values of $\alpha$, unstable states are found only in a limited range of values of the partial pressures. This stays in contrast to what happens when $\alpha=0.25$ and the experimental findings, 


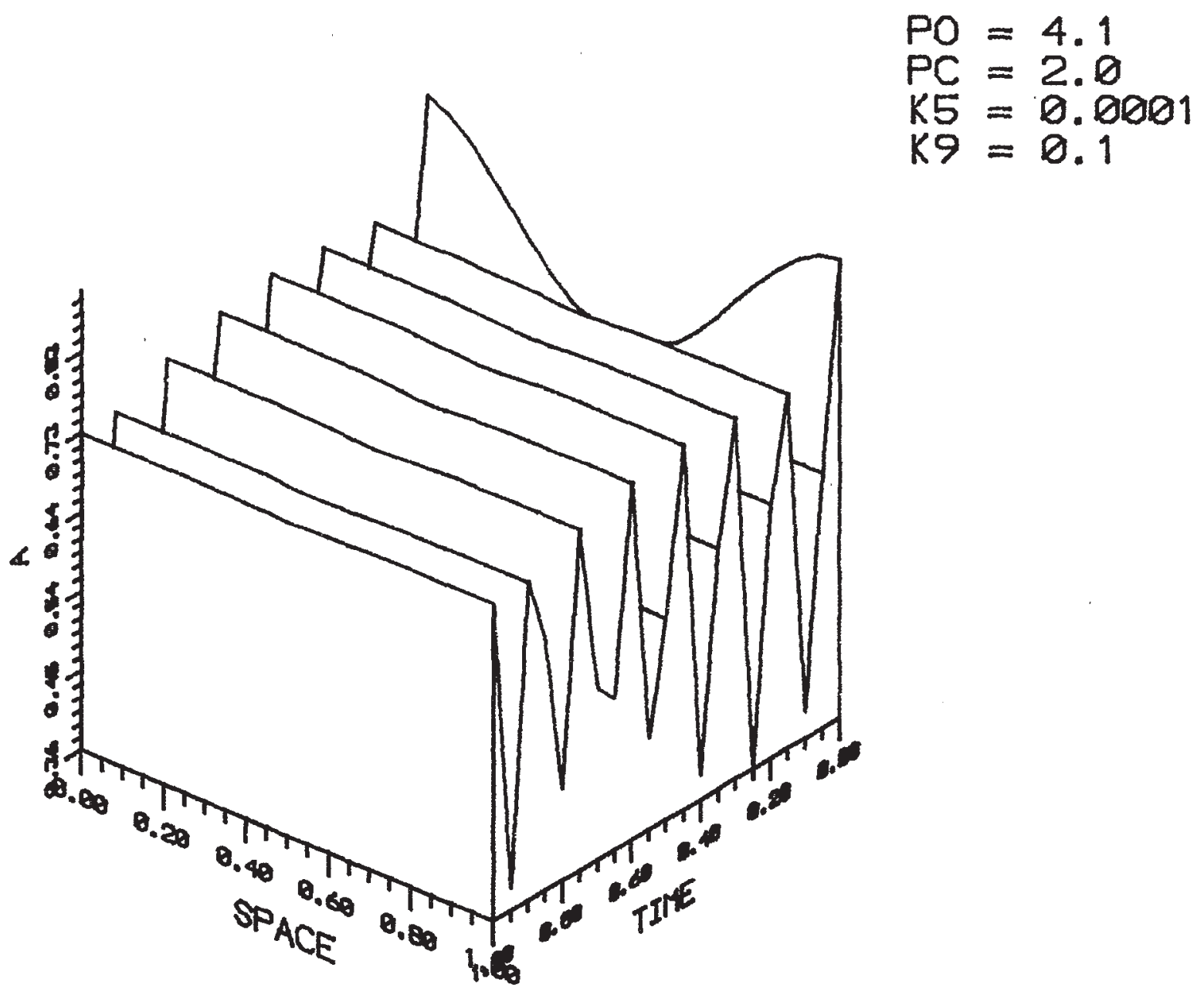

FIG. 4. Surface time-space evolution of the variable $a$, when $\left(P_{c}, P_{o}\right)=(2,4.1)$. The variation of space and time is the same as in Fig. 2 .

which indicate unbounded instability regions when both $p_{c}$ and $p_{0}$ grow.

\section{NUMERICAL RESULTS FOR THE HOMOGENEOUS SURFACE}

In this section we discuss the results obtained for a system with uniform properties, i.e., the variables do depend upon the site on the surface, but the properties of each site are the same. We have numerically integrated the nonlinear systems $S 1$ and $S 2$ using a finite difference method to treat the space dependence and a Runge-Kutta routine to account for the time progression. The length of the catalyst was divided into discrete cells (maximal 100). The initial conditions are either gathered by imposing a periodic perturbation or random fluctuations. We have considered either periodic (infinite sample) or no flux boundary conditions.

The analysis of the space independent problem ${ }^{19}$ has shown that the detailed behavior of the phase diagram in the $P_{c} \times P_{o}$ plane is very rich, with a very large number of fixed points and several different types of bifurcations. The situation is much the same for the space dependent problem and a detailed exploration of the diagram is very lengthy. Therefore, we have focused our attention on some characteristic points within the instability region of the diagram, trying to identify some major aspects of the transition from the uniform solutions to the dissipative structures.

As in the linear study, the results we obtain for the full system also depend upon the values taken for the diffusion coefficients. With the realistic values there will be no changes with respect to the situation of the homogeneous problem: the instability region is characterized by the homogeneous oscillating surface, although the bifurcation which the system undergoes to cross the instability line may be of several different types.

For the purpose of discussing a richer situation we have carried out an investigation for the same values of the diffusion coefficients used in the linear study. The values of the other constants are shown in Table I and we integrated the 

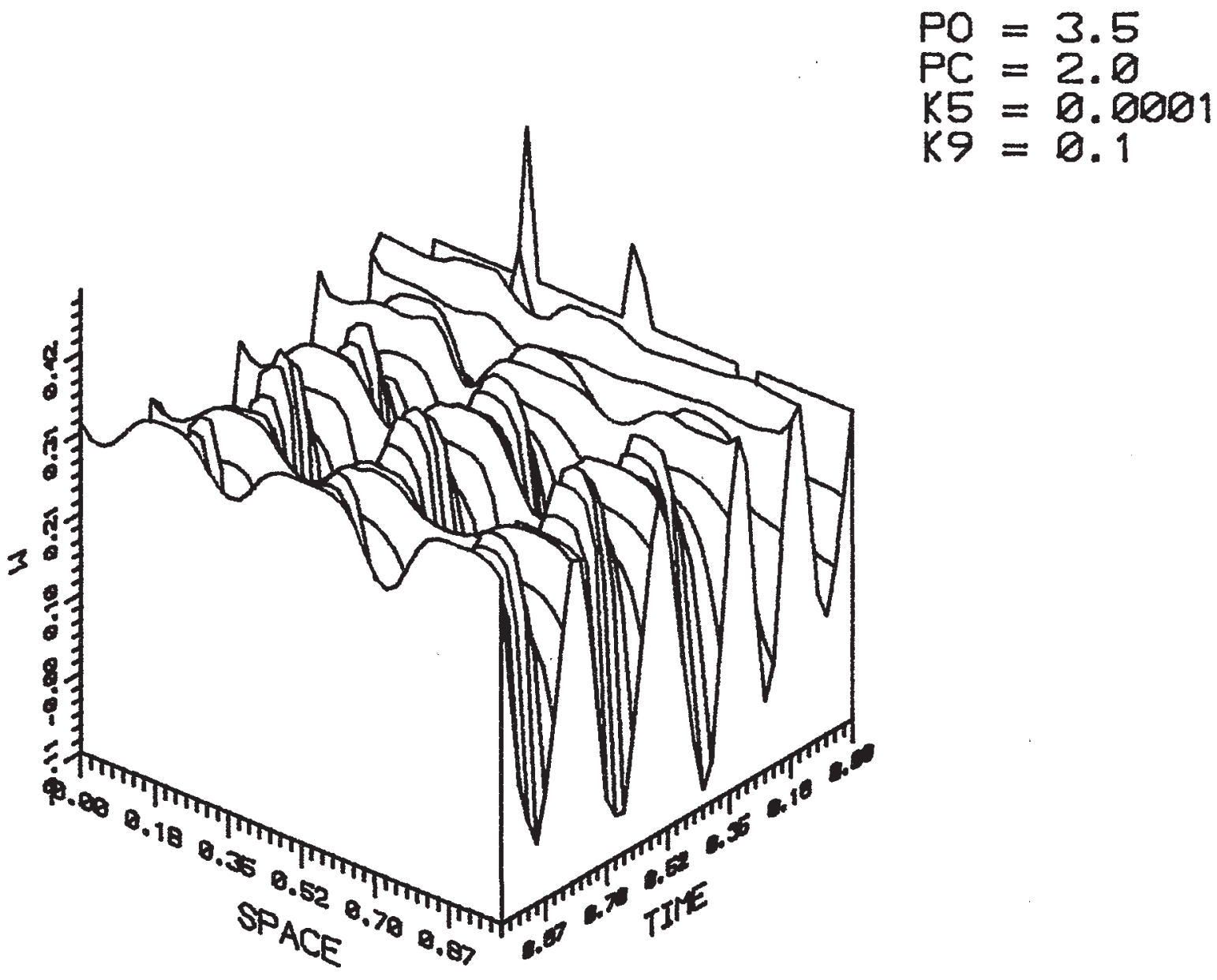

FIG. 5. Surface time-space evolution of $w(S 2)$ when $\left(P_{c}, P_{o}\right)=(2,3.5)$. The length of the sample is $0.35 \mathrm{~cm}$ and the total time of integration is 50 seg.

system for a series of $\left(p_{c}, p_{o}\right)$ points. Let us now discuss the features of the solutions for those points indicated in Fig. 1, which are typical of several other investigated points. Though the actual value of $k_{4}$ (used in the linear stability analysis) is very large $\left(5 \times 10^{5}\right)$, we have taken a smaller value in the integration of $S 1\left(10^{2}\right)$, otherwise the time step becomes very small, increasing the CPU time. This change causes only small deviations in the locus of the transition line.

For $P_{c}=2$, the point $P_{o}=3.1$ lies just above the Hopf instability line, where uniform oscillations were observed in the space independent model. Now however, as foreseen by the linear stability analysis there is a transition from the uniform state to a stationary Turing structure as shown in Fig. 3, where the initial condition consisted of a local disturbance superimposed on the uniform reference state. Varying the size of the system we have also checked that the wavelength is intrinsic.

On increasing $P_{o}$ to 4.1 , even if one starts from modu- lated initial conditions, the system evolves to uniform oscillations (Fig. 4).

In between, for instance for $P_{o}=3.5$ (Fig. 5), we detected a region of mixed-mode Turing-Hopf solutions where the amplitude of the Turing structures oscillates periodically in time. This succession of solutions is not unexpected as in this region of the $P_{o} \times P_{c}$ plane the Turing and Hopf instabilities of the uniform steady solution are very close (near codimension 2 situation). It is then well known that the interaction between the Turing and Hopf modes leads to nontrivial spatiotemporal behavior. ${ }^{22}$

In the precise case discussed here we recover one of the bifurcation diagrams, sketched in Fig. 6, characteristic of such a situation. In this scenario a stable Turing structure appears first at the values $P_{o}^{T}$ corresponding to the dashed line in Fig. 1. The Hopf oscillations that bifurcate at $P_{a}^{H}$ (plain line in Fig. 1) are destabilized until $P_{0}^{H^{\prime}}$ by the existence of the spatial modes. The Turing structures themselves 


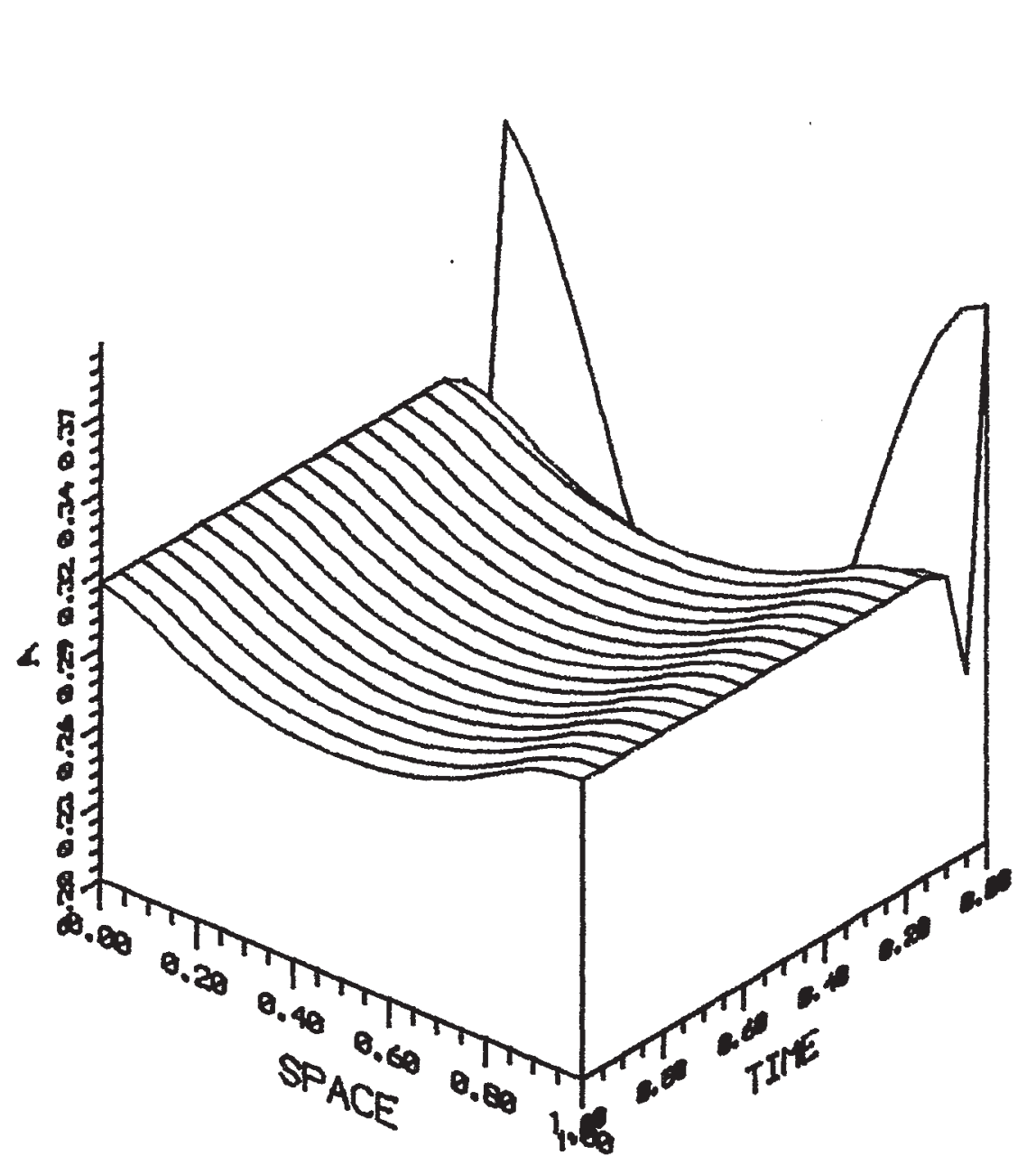

FIG. 6. At the border of the instability region $\left(P_{c}, P_{0}\right)=(3,14.8)$ the static pattern recovers stability.

become unstable at $P_{0}^{T^{\prime}}$ with respect to the formation of a branch of stable mixed modes that links the Turing and Hopf branches.

For all $P_{o}>P_{o}^{H^{\prime}}$, the uniform oscillations are the sole stable solutions as has been verified by numerical computations, until one nears the upper boundary line in the stability diagram (Fig. 1) where another Turing bifurcation occurs, interacting now with oscillations generated by a SNIPER bifurcation. ${ }^{23}$ Indeed in Fig. $7\left(P_{c}=3, P_{o}=14.8\right)$ the Turing pattern is again stable.

The results of this section show several different types of dissipative structures, corroborating our claims that the instability diagram is indeed very rich. However, they point towards the nonexistence of traveling wave solutions to the surface homogeneous systems $S 1$ and $S 2$. For any value of the diffusion coefficients both linear and nonlinear investigations failed to detect them, though we have taken several different values for $k_{5}$ and $k_{9}$ and investigated many qualitatively different regions of the diagram, as those close to the

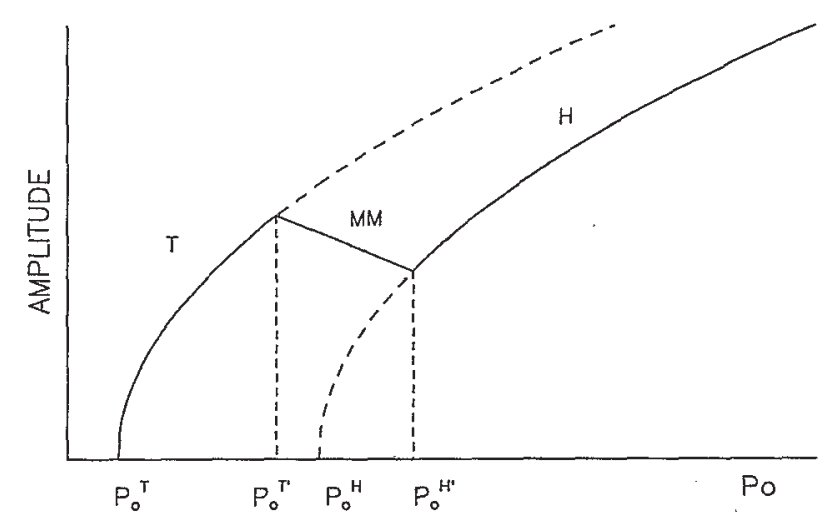

FIG. 7. Schematic diagram for the Turing and Hopf amplitudes close to a codimension 2 bifurcation. Full/dashed line indicates stable/unstable situation. $T, H$, and $M M$ indicate, respectively, Turing, Hopf, and mixed modes. 

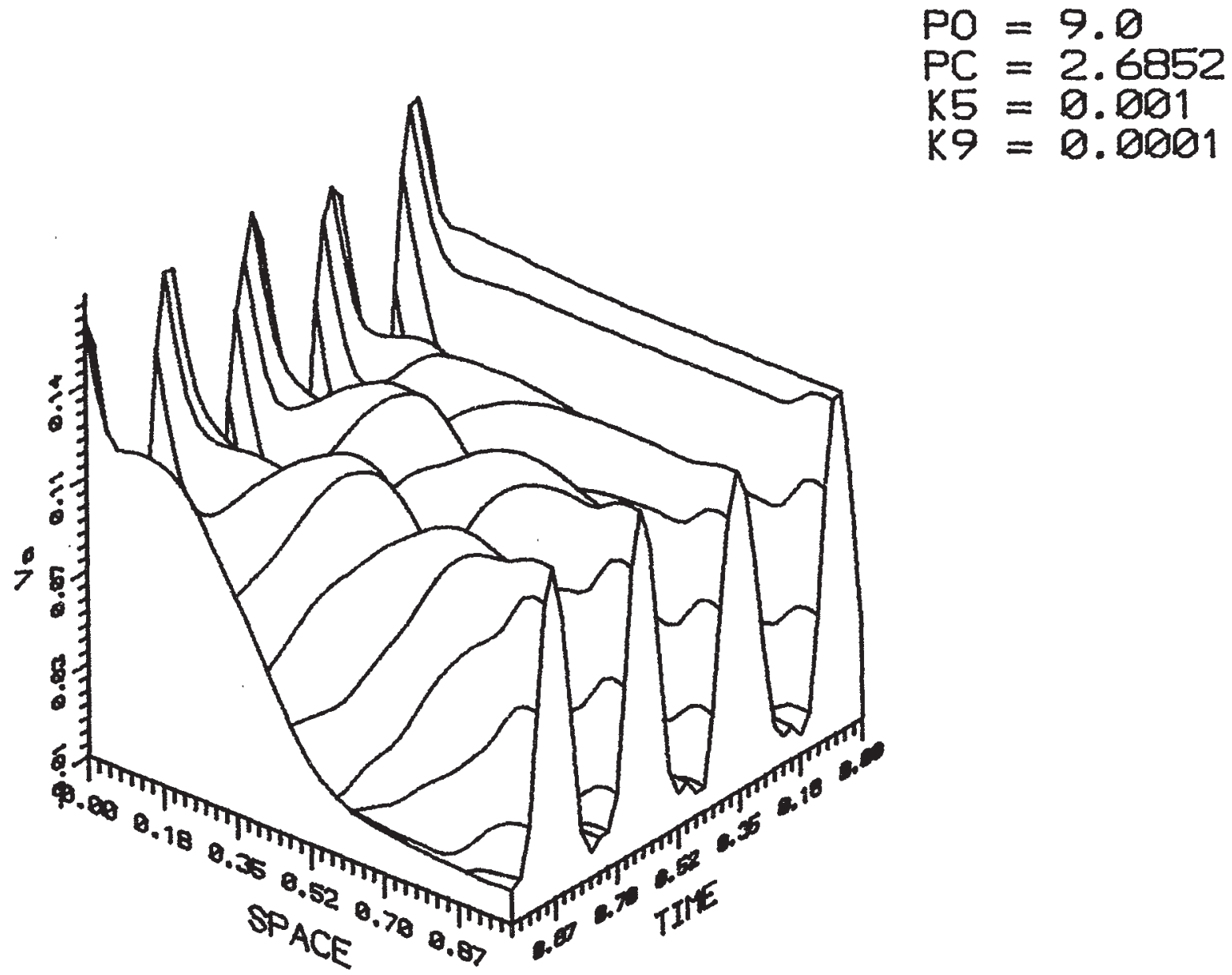

FIG. 8. Wave propagation when the first cell has a different defect rate ( $\alpha$ ) than all of the others. The cells are unstable for both values of $\alpha$.

instability lines of Hopf and saddle-node bifurcation and also points deep inside the instability region.

However, the occurrence of this pattern is a well defined behavior of the system which have been clearly exposed in the experiments. The fact that they were not found for a homogeneous surface supports the conjecture raised up by experiments that inhomogeneities in the surface act as seeds to nucleate the formation of the waves. In Sec. V we discuss the results obtained as we let the surface properties be site dependent.

\section{THE INHOMOGENEOUS SURFACE}

Now it is assumed that the defect rate $\alpha$ may be different from cell to cell for the reasons discussed before. Thus each cell has its own linear eigenfrequency. For instance take the point $\left(P_{c}, P_{o}\right)=(2.2,6.5)$. The integration of the kinetic equations for the space independent model shows the following dependence for the periods of oscillation as a function of $\alpha: T(0.4)=4.7 \mathrm{~s} ; T(0.25)=7.4 \mathrm{~s} ; T(0.15)=8.8$ s. Different periods of oscillation in neighboring cells appears as a basic mechanism for wave propagation. Indeed, each cell suffers the influence of its neighbors through the diffusion terms, and this coupling alters its own period of oscillation. As there is a certain delay in the response to this influence, neighboring cells will oscillate out of phase, resulting, as overall picture, in a wave propagation pattern. A more subtle situation which we also discuss below occurs when one cell is stable and its neighbor unstable.

The wave propagation pattern is also very sensitive to the values of the diffusion coefficients. If they are large they couple the cells very strongly, and the picture sketched above may be replaced by a homogeneous oscillating pattern. So we present results obtained for small values of $k_{5}\left(=10^{-3}\right.$ $\left.\mathrm{cm}^{2} / \mathrm{s}\right)$ and $k_{9}\left(=10^{-4} \mathrm{~cm}^{2} / \mathrm{s}\right)$. Since $k_{5} / k_{9}=10$ we see that static Turing structures are excluded from the possible solutions. From a qualitative point of view the situation here analyzed is similar both to the one obtained with realistic 

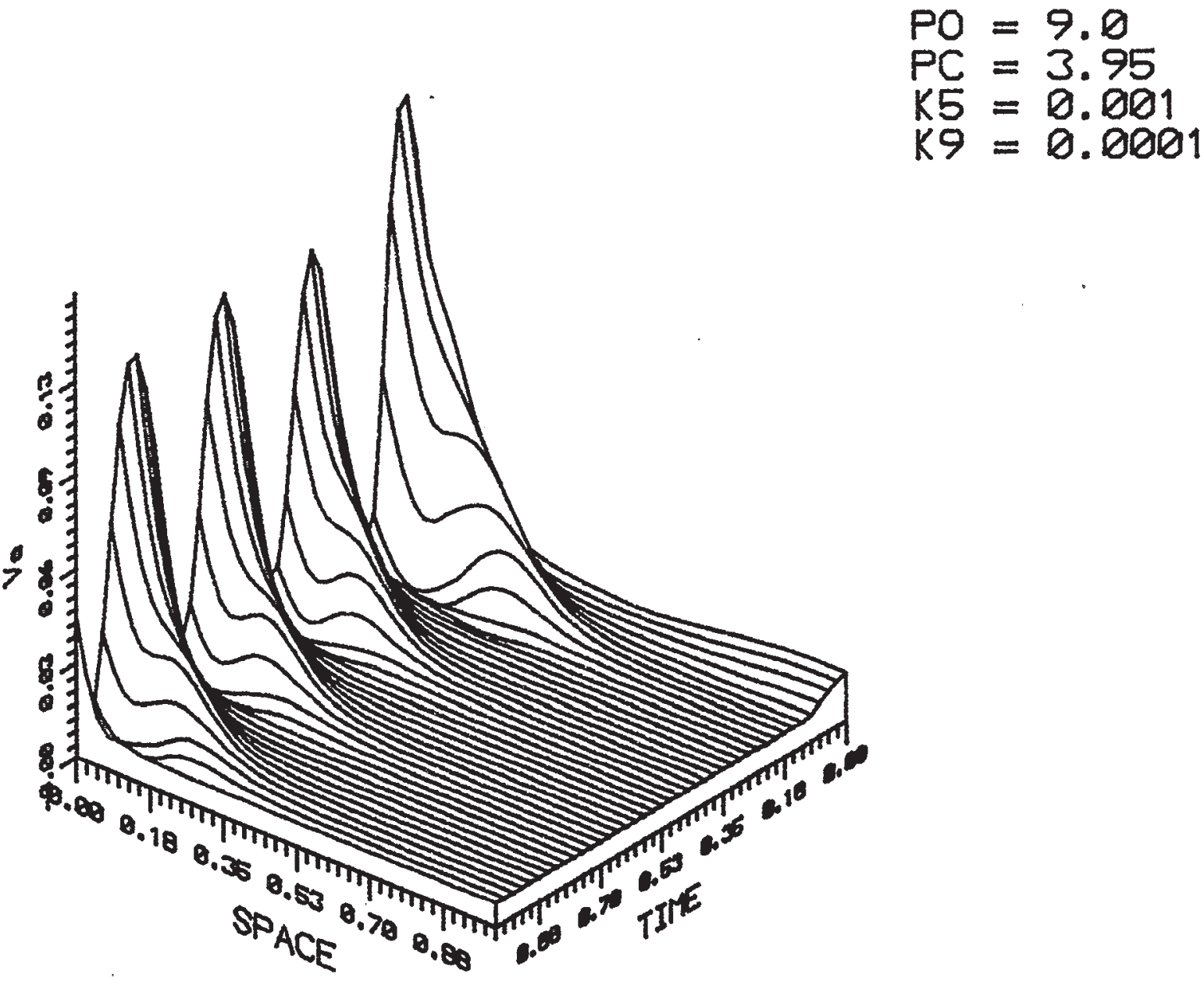

FIG. 9. Damped wave propagation, when the first cell is unstable but all of the others are stable.

values of $k_{9}\left(\sim 10^{-15}\right)$ and also to the one investigated in Ref. 17 , where $k_{9}=0$.

Figures 8-11 show several situations for the points of the diagram indicated in Fig. 2. $\alpha$ takes the value 0.15 for all but the first site, where $\alpha=0.4$. It is seen that wave propagation settles in different situations. The best examples occur when all sites are unstable (Fig. 8). As discussed before, the cell with highest defect rate (smallest period) triggers the perturbation, and since the neighboring cells are also unstable, it is propagated down the sample with almost constant amplitude. If only the first site is unstable the wave propagation will not be complete: in each cell the amplitude of the perturbation will be damped, and the excitation of the next cell will be smaller. If the cells are about to become unstable, the damping is small and the waves may even reach the other end of the sample, but with a smaller amplitude. Otherwise the perturbations will be wiped out and will not cover the whole path (Fig. 9).

We have also investigated the situation where the end site is stable but the inner are unstable (Fig. 10). Though this is not always thought as a possible physical realization, we can see by the instability diagrams (Fig. 2) that this indeed corresponds to a very common situation. The integration shows a kind of signal propagation, which actually corresponds to the damping of first site that is propagated to the neighboring sites, but whose effect is gradually lost when it moves away from the stable cell. The end sites oscillate almost in phase.

Finally we illustrate with Fig. 11 the situation when the first and last sites have different $\alpha$ 's as those of the interior of the sample. As expected, it shows competition between waves triggered from both extremities.

\section{CONCLUSIONS}

In this work we investigated the occurrence of spatiotemporal dissipative structures during the oxidation of $\mathrm{CO}$ on $\mathrm{Pt}(100)$ by the analysis of a system of reaction-diffusion 

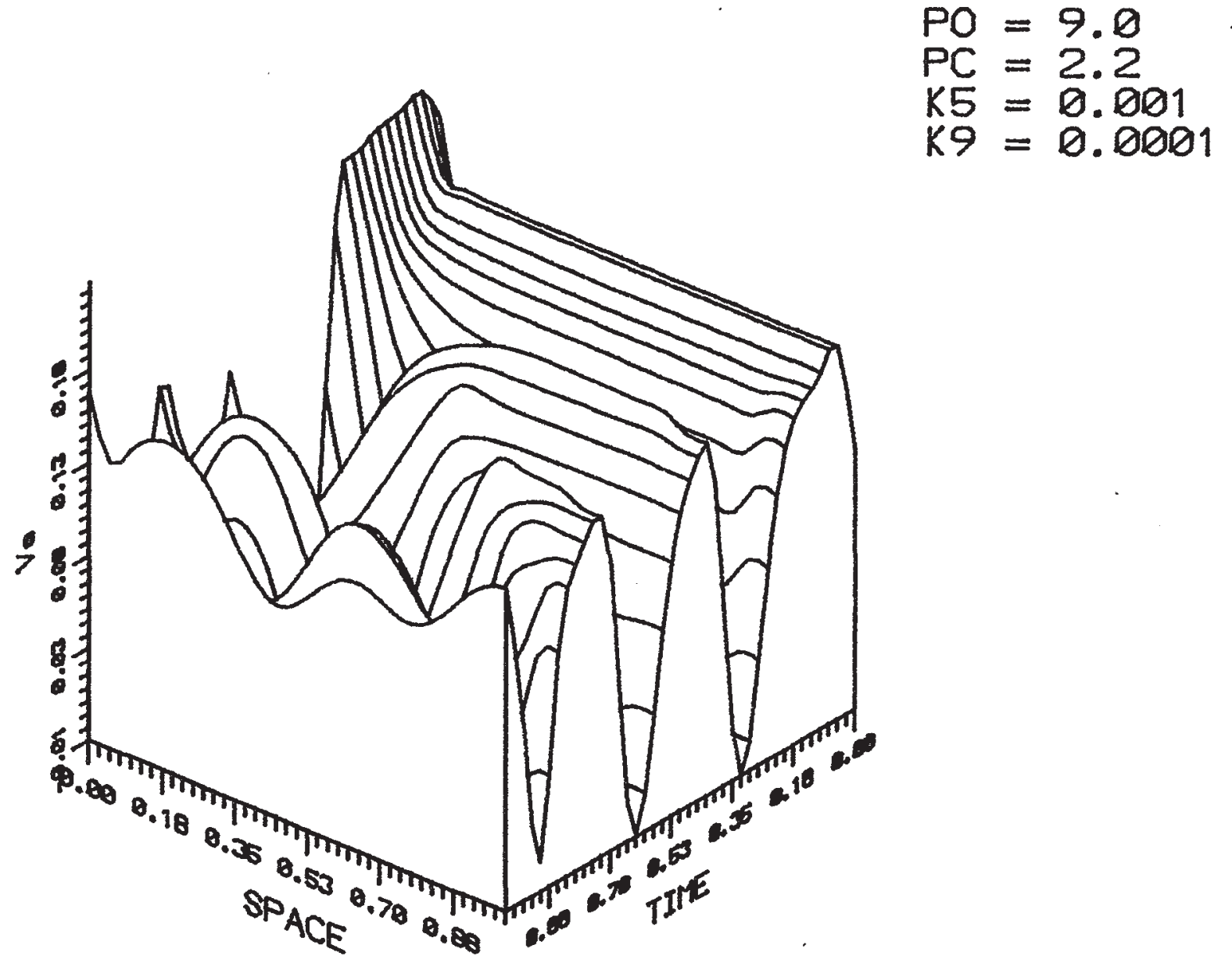

FIG. 10. The situation here is reversed in comparison to Fig. 9. The first cell is stable, while all others are unstable. Note a kind of damping propagation from left to right.

equations. This problem and the CO oxidation on $\mathrm{Pt}(110)$ have been the object of intense investigation, both in the homogeneous as in the space dependent situation, here with the help of cellular automata and also of reaction-diffusion systems. ${ }^{24-26}$ The form of the system reflects the basic mechanisms of this phenomenon, which have long been identified. Nevertheless this work introduces a new diffusion-like term, related to interface energy between patches of different crystalline order which may be found on the $\mathrm{Pt}(100)$ surface. It stems in a quite natural way from the site interaction model for the surface energy. The value of this diffusion coefficient is much smaller than that of the normal diffusion of the adsorbed species, and this is a support for neglecting it if realistic values for all constants are introduced in the model. However, the presence of this new term opens the possibility of a much richer class of solutions, whose features will depend, in a crucial way, on the ratio of the two diffusion coefficients.

Besides this new observed dependence, it is well known that the behavior of the system depends on whether we consider a homogeneous or inhomogeneous surface. In the first case we have shown that, with realistic values of the diffusion coefficients, the system has only homogeneous oscillations. On the other hand, if we take much larger values of the new diffusion coefficient, we note the presence of Turing modes and the much rarer mixed Turing-Hopf mode.

The modeling of space inhomogeneous situations amounts to assigning different values of some of the model parameters to different surface sites. In this work we supposed that the parameter $\alpha$, related to the defects rates on the surface, takes different values depending on whether the site is on the edge or in the interior of the sample. This procedure causes different stability properties to the sites. Depending on the values of $\alpha$ and of the partial pressures we distinguish three situations: (i) both sites are stable; (ii) both are unstable; (iii) one is stable and the other unstable. We have found that the experimentally observed wave propagation, according to experimental observations, settles when the dif- 

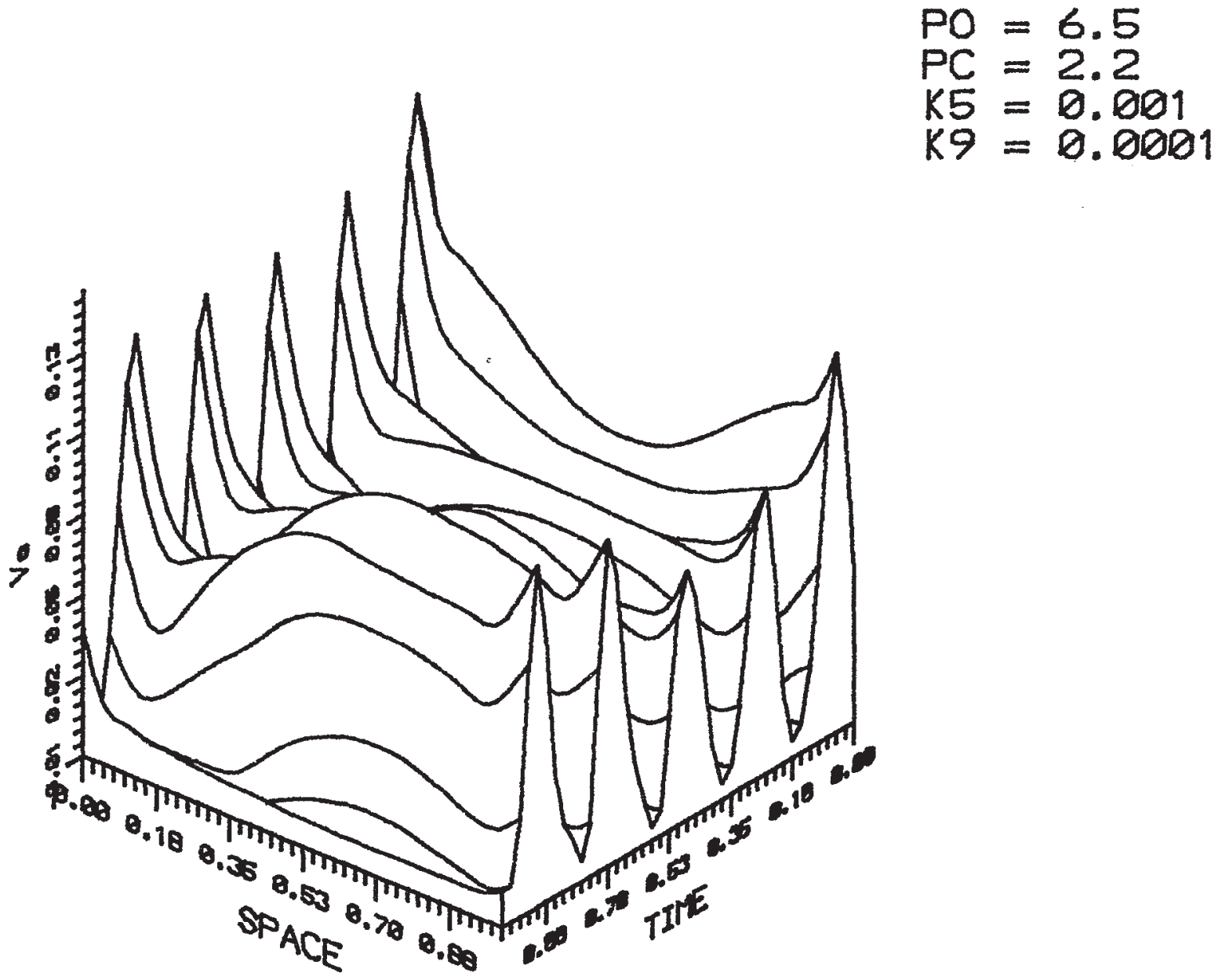

FIG. 11. Situation where both extremities act as trigger zones. In the case, the first site (at the left) has a greater value of $\alpha(0.4)$ than the last site $(0.25)$ and the wave it triggers settles in.

ferent sites are unstable. Its origin may be understood as the result of different oscillation periods, whereas the site with highest period (also the highest defect rate) acts as pacemaker, causing an increase of the period of the neighboring cell, this process being repeated from cell to cell. In case (iii) wave propagation is also observed, but, depending on the value of the parameters, it may be damped in its way along the sample.

To close this discussion we stress that our main results, absence of waves in the homogeneous surface and their presence in the inhomogeneous situations where the role of pacemakers is played by the high defect cells, corroborate both the previous theoretical study (using a set of coupled diffusion-reaction equations) and the experimental investigations, and contrast to those obtained by cellular automata, which indicate wave fronts even in a homogeneous surface. The extension of this work to two-dimensional surface is a matter of some extra computational effort. Preliminary results $^{27}$ indicate the same kind of behaviors as for the one- dimensional situation, whereby a richer Turing and wave propagation structures may be found.

${ }^{1} \mathrm{G}$. Nicolis and I. Prigogine, Self-Organization in Nonequilibrium Systems (Wiley, New York, 1977).

${ }^{2}$ L. F. Razon and R. A. Schmitz, Catal. Rev. Sci. Eng. 28, 89 (1986).

${ }^{3}$ R. Imbhil, in Optimal Structures in Heterogeneous Reaction Systems, edited by P. J. Plath (Springer, Berlin, 1989), p. 26.

${ }^{4} \mathrm{G}$. Ertl, Science 254, 1750 (1991).

${ }^{5}$ M. Eiswirth, K. Krischner, and G. Ertl, Appl. Phys. A 51, 79 (1990).

${ }^{6} \mathrm{G}$. Ertl, in Spatial Inhomogeneities and Transient Behaviour in Chemical Kinetics, edited by P. Gray et al. (Manchester University, Manchester, 1990), p. 565.

${ }^{7}$ R. Imbhil, M. P. Cox, and G. Ertl, J. Chem. Phys. 84, 3519 (1986).

${ }^{8}$ M. Eiswirth and G. Ertl, Surf. Sci. 177, 90 (1986).

${ }^{9}$ M. Eiswirth, P. Möller, K. Wetzl, R. Imbhil, and G. Ertl, J. Chem. Phys. 90, 510 (1989).

${ }^{10}$ M. P. Cox, G. Ertl, and R. Imbhil, Phys. Rev. Lett. 54, 1725 (1985).

${ }^{11}$ H. H. Rotermund, S. Jakubith, A. von Oertzen, and G. Ertl, J. Chem. Phys. 91, 4942 (1989)

${ }^{12}$ M. E. Kordesh, W. Engel, G. Lapeyere, E. Zeitler, and M. Bradshaw, Appl. Phys. Lett. A 49, 399 (1989). 
${ }^{13}$ J. Falta, R. Imbhil, and M. Henzler, Phys. Rev. Lett. 64, 1409 (1990). ${ }^{14}$ S. Jakubith, H. H. Rotermund, W. Engel, A. von Oertzen, and G. Ertl, Phys. Rev. Lett. 65, 3013 (1990).

${ }^{15}$ H. H. Rotermund, W. Engel, M. Kordesh, and G. Ertl, Nature 343, 355 (1990).

${ }^{16}$ P. Möller, K. Wetzl, M. Eiswirth, and G. Ertl, J. Chem. Phys. 85, 5328 (1986).

${ }^{17}$ R. Imbhil, M. P. Cox, G. Extl, H. Müller, and W. Brennig, J. Chem. Phys. 83, 1578 (1985).

${ }^{18}$ R. F. S. Andrade, in Spatial Inhomogeneities and Transient Behaviour in Chemical Kinetics, edited by P. Gray et al. (Manchester University, Manchester, 1990), pp. 741-743.

${ }^{19}$ R. F. S. Andrade, G. Dewel, and P. Borckmans, J. Chem. Phys. 91, 2675 (1989).
${ }^{20}$ G. T. Rado and H. Suhl, Magnetism (Academic, New York, 1963), Vol. 1. ${ }^{21}$ B. Poelsema, S. T. de Zwart, and G. Comsa, Phys. Rev. Lett. 49, 578 (1982).

${ }^{22}$ H. Kidachi, Prog. Theor. Phys. 63, 1152 (1980).

${ }^{23} \mathrm{~J}$. Guckenheimer and P. Holmes, Nonlinear Oscillations, Dynamical Systems and Bifurcation of Vector Fields, Appl. Math. Sci. 42 (Springer, Berlin, 1983).

${ }^{24}$ K. Krischer, M. Eiswirth, and G. Ert1, J. Chem. Phys. 97, 307 (1992).

${ }^{25}$ M. Bär, Ch. Zülicke, M. Eiswirth, and G. Ertl, J. Chem. Phys. 96, 8595 (1992).

${ }^{26}$ M. Bär, M. Falcke, H. Engel, and M. Eiswirth, J. Chem. Phys. 97, 4555 (1992).

${ }^{27}$ D. Lima, M.Sc. thesis, Universidade Federal da Bahia, Salvador, Brazil (1993). 